\title{
Strain-Induced Diffusion of Nickel in Nanocrystalline Fe Produced by High Energy Shot Peening
}

\author{
Sun Jianchun, Chen Dengming, Ma Yilong \\ Chongqing University of Science and Technology, Chongqing 401331, China
}

\begin{abstract}
A nanocrystalline surface layer without oxidation, porosity or contamination was obtained by high energy shot peening (HESP) to a pure iron cylinder. The thickness of the nanocrystalline surface layer was more than $100 \mu \mathrm{m}$ and the average grain size was about $50 \mathrm{~nm}$. The pulse pressure diffusion (PPD) and constant pressure diffusion (CPD) were used to diffuse nickel into nanocrystalline iron at $850^{\circ} \mathrm{C}$ on Gleeble 1500 . Results show that the diffusion coefficient of $\mathrm{Ni}$ in the nanocrystalline $\mathrm{Fe}$ is 1 order of magnitude higher than that in coarse-grained Fe and 1 order of magnitude higher than that using CPD. The enhanced diffusivity of $\mathrm{Ni}$ may originate from the following three reasons: (1) pulse pressure can break the metallic compound formed during diffusing which may block the diffusion of $\mathrm{Ni}$ to inner nanocrystalline $\mathrm{Fe}$; (2) a considerable amount of triple junctions and (3) a large volume fraction of non-equilibrium grain boundaries (GBs) in the present nanocrystalline Fe sample processed by the HESP technique.
\end{abstract}

Key words: nanocrystalline Fe; high energy shot peening; diffusion coefficient; nickel atom

During the fabrication and treatment of materials, such as nitridation, surface alloying and diffusion bounding, an enhanced diffusivity of atoms at low temperature and pressure is always wanted. According to the diffusion theory, to enhance the diffusion coefficient at a fixed or lower temperature, there must be more diffusion channels in solids. The difference between nanocrystalline materials and the conventional polycrystalline systems is the increased volume fraction of grain boundary region which makes the nanocrystalline materials to exhibit, in general, an enhanced atomic diffusion. It has been reported by the experimental results in the literature ${ }^{[1-3]}$. However nanomaterials prepared by current methods, such as gas phase condensation, mechanical alloying and sputtering, always contain impurities and holes, which may hinder the diffusion of atoms in them. So bulk nanocrystalline solids without porosity and contamination are always expected to be prepared for the study of the diffusion of atoms in nanocrystalline materials.

Because the atomic diffusion in nanocrystalline is mainly the grain-boundary diffusion ${ }^{[4-7]}$, one main direct way to enhance the diffusivity of atoms in them is refining the grains to increase the fraction of grain boundaries, which can act as diffusion channels. But to a fixed nanomaterial, loading a pressure on the diffusion couple can help to improve the movement speed of the atoms of diffusion source and an atomic level contact of the diffusion couples. Here two different pressures, constant pressure and pulse pressure, were loaded on the iron and nickel diffusion couple, and the diffusion processes were called constant pressure diffusion (CPD) and pulse pressure diffusion (PPD) respectively. Due to a high activation of atoms in nanocrystalline materials, when the atoms exchanged from two different materials into each other, metallic compounds always formed on the interface, which on some extend would prevent the further diffusion of atoms ${ }^{[8-10]}$. A pulse pressure exerted on the xenogenic metallic materials has been proved to be an effective way to delete the metallic compound formed during the diffusion ${ }^{[11]}$. In the present work, the iron-nickel diffusion couples were subjected to a pulse pressure to enhance the diffusivity of nickel atoms in iron, and we defined the diffusion as strain-induced diffusion.

Foundation item: National Natural Science Foundation of China (51201191); Scientific and Technological Research Program of Chongqing Municipal Education Commission (KJ1401329); Cooperative Project of Academician Workstation of Chongqing University of Science \& Technology (CKYS2014Y01); Research Foundation of Chongqing University of Science \& Technology (CK2013B09)

Corresponding author: Sun Jianchun, Ph. D., Associate Professor, College of Metallurgy and Materials Engineering, Chongqing University of Science and Technology, Chongqing 401331, P. R. China, Tel: 0086-23-65023714, E-mail: kwensun@163.com 


\section{Experiment}

An iron cylinder ( $\Phi 10 \mathrm{~mm}$ and $\Phi 30 \mathrm{~mm}$ ) with a purity of 99.95\% and homogeneous coarse grains of $\alpha$-Fe phase (100 $\mu \mathrm{m}$, in Fig.1) was subjected to the high energy shot peening (HESP) processing to get a nanocrystalline surface layer. Before HESP treatment the $\mathrm{Fe}$ sample was polished and fully annealed at $800{ }^{\circ} \mathrm{C}$ in a vacuum heat treatment furnace for $200 \mathrm{~min}$ to eliminate the effect of inner stress and mechanical polishing on the surface structure.

During the HESP procedure, hardened cast iron balls (with a mirror-like surface and a diameter of $0.5 \mathrm{~mm}$ ) were placed at the bottom of shot tank (Fig.2). When the compressed gas was blown into the working tanker from the peening gun, the balls were accelerated and got a high kinetic energy. After the balls with a high kinetic energy reached the surface of the sample, the kinetic energy would be transformed into plastic deformation in the samples. The balls after the release of energy were absorbed into the gun again because of a negative pressure and this made the HESP procedure recycling, which would lead to an accumulation of deformation in the samples. The accumulated deformation would result in a severe deformation in the sample, and it may cause grain refinement of the sample.

Microscopy and SEM were used to examine the microstructure features of the nanocrystalline layers. Meanwhile EDS was used to determine the diffusion depth and Ni contamination in nanocrystalline Fe. The X-ray diffraction (XRD) was used to study the structure evolution of the layer of the Fe sample with $\mathrm{Cu} \mathrm{K} \alpha$ radiation. Small angular steps $2 \theta=0.03^{\circ}$ were taken to measure the intensity of each Bragg diffraction peak. The counting time of $20 \mathrm{~s}$ was used to measure the width of diffraction peak in the step-scanning mode. The average grain size and the mean microstrain of the nanocrystalline Fe layer were derived from the line broadening of BCC Fe (110), (200) and (211) Bragg diffraction peaks by Scherrer method.

The diffusion of nickel in nanocrystalline and coarse Fe was carried on the Gleeble-1500D thermal simulation machine which can provide a controllable temperature and pressure in a vacuum environment. To compare the diffusion of nickel in both nanocrystalline and coarse $\mathrm{Fe}$, we designed an assembly of diffusion samples, as shown in Fig. 3, which can provide the

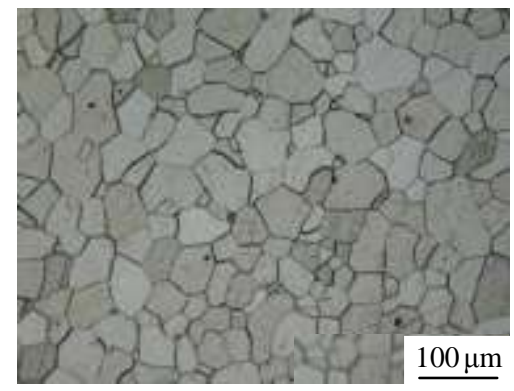

Fig. 1 Metallurgical structure of iron after annealing

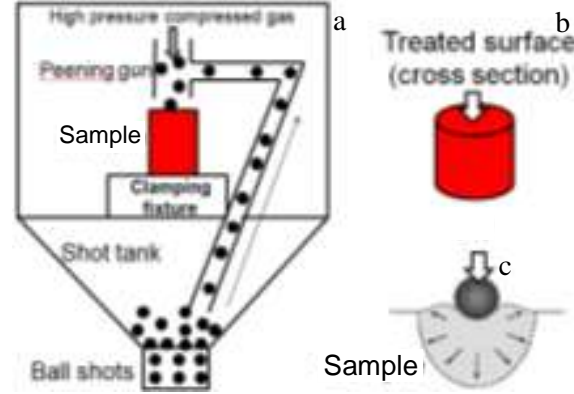

Fig.2 Schematic illustration of the HESP treatment set-up

same diffusion parameters for nickel in both nanocrystalline and coarse Fe.

Pure nickel foil with a thickness of $13 \mu \mathrm{m}$ was used as the diffusion source, see in Fig.3. The diffusion was conducted at $850^{\circ} \mathrm{C}$ within 20 min under a constant pressure $(\mathrm{CP}, F=10$ $\mathrm{MPa}$ ) and a pulse pressure (PP, $\left.F_{\max }=16, F_{\min }=8, f=0.5 \mathrm{~Hz}\right)$.

\section{Results and Discussion}

\subsection{Microstructure characterization of the HESPed Fe}

Detailed microstructure of the surface layer was characterized by SEM observation of the cross-section of the peened samples. Fig. 4 shows the full view of HESPed Fe after 7 min HESP treatment and the high magnification of the top surface layer. As shown in Fig.4, the whole deformed layer with a thickness about $120 \mu \mathrm{m}$ contain three different parts: the severely deformed part on the top surface in which the grains are extremely refined (marked as A), the deformed layer in which the grains are refined to some extent (marked as B) and the transitional region in which the grains are yet not refined and just a little deformation occurs (marked as C). But there are no obvious interfaces between the 3 regions, so here we call the whole deformed layer as HESPed layer. As shown in the high SEM magnification of the top surface layer, the grains cannot be separated from each other because there are no obvious boundaries of the iron, which indicates HESP treatment can refine the iron grains. Besides the grain orientation has been changed and fiber structure forms because of HESP treatment, but whether the grains are nanosized or not is uncertain. The

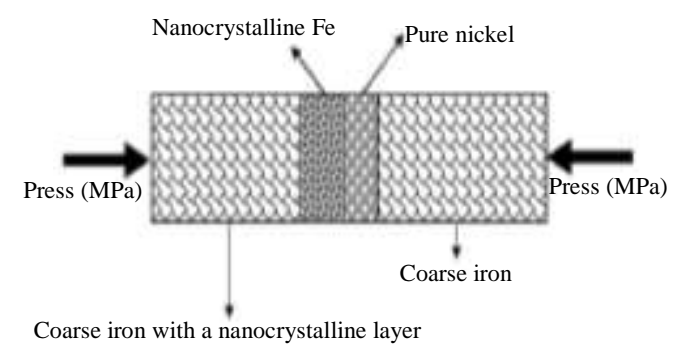

Fig.3 Assembly of diffusion samples 


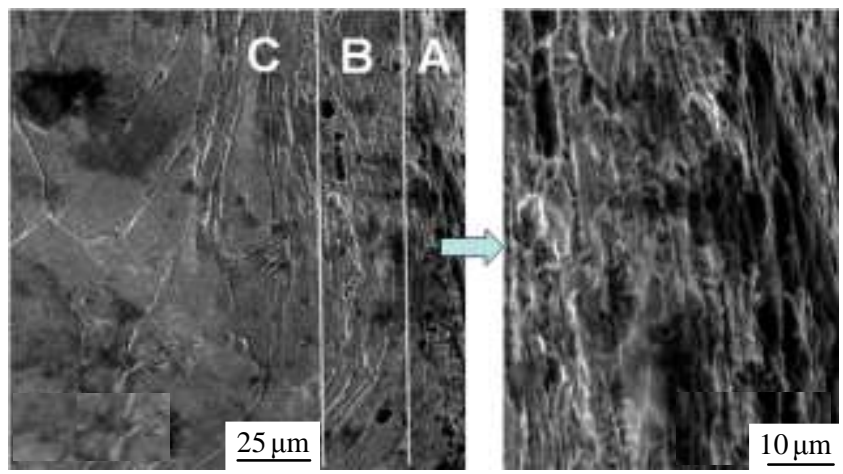

Fig.4 Cross-section SEM image of the sample peened for $7 \mathrm{~min}$

$\mathrm{XRD}$ analysis was used to determine whether the grain size of the top surface layer reaches nano-size.

Fig. 5 shows the XRD scans of the top surface layer of the samples peened by different time. As shown in Fig.5, the XRD peaks of the layers appear at the same $2 \theta$ position, which mean that there are no phase transformation during the HESP treatment on iron. But by contrast to the pre-treated samples, the diffraction peaks of the HESPed samples obviously broaden, suggesting that the grains of the iron are refined or a large microstrain appear in the iron after HESP treatment.

The average grain size $(D)$ and the mean microstrain of the nanocrystalline $\mathrm{Fe}$ were derived from the line broadening of $\mathrm{Fe}$ Bragg diffraction peaks according to the Scherrer method:

$$
D=\frac{k \lambda}{m \cos \theta}
$$

If the grain refining and microstrain coexist in a crystalline material, the half width of the diffraction profile broadens, grain size and microstrain will have the following relationship:

$$
\frac{F W(s) \cos (\theta)}{\lambda}=\frac{K}{D}+4 \varepsilon \frac{\sin \theta}{\lambda}
$$

Table 1 lists the grain size and microstrain of the top surface of the sample peened from $3 \mathrm{~min}$ to $10 \mathrm{~min}$ calculated by Eq.(2). And with the increase of the HESP treatment time, the grain size of the nanocrystalline iron layers is further refined, but with a rise of microstrain.

\subsection{Determination of diffusion coefficient}

Concentration and diffusion distance of nickel in samples after diffusion were determined by EDS.

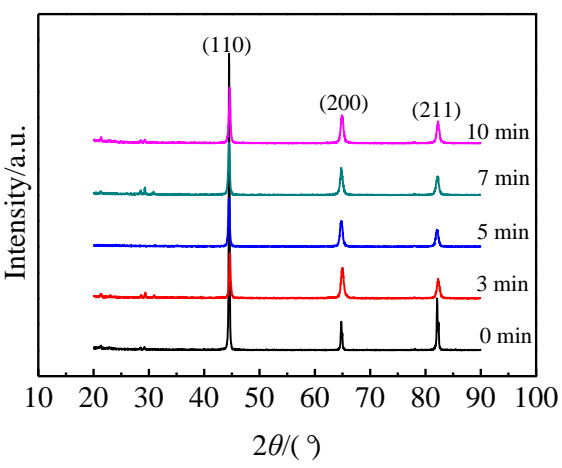

Fig.5 XRD patterns of sample's surface processed within different duration time

Table 1 Characterization of the nanocrystallized iron layers prepared by HESP

\begin{tabular}{ccc}
\hline Shot time/min & Grain size/nm & Microstrain/\% \\
\hline 3 & 58.5 & 0.0412 \\
5 & 49 & 0.0477 \\
7 & 27.4 & 0.0423 \\
10 & 21.2 & 0.0486 \\
\hline
\end{tabular}

The nickel diffuses in $\mathrm{Fe}$ in this paper with the following boundary conditions:

When $t=0,\left\{\begin{array}{l}C=C_{0}, x>0 \\ C=0, x<0\end{array}\right.$
When $t>0,\left\{\begin{array}{l}C=C_{0}, x=-\infty \\ C=0, x=\infty\end{array}\right.$

According to the boundary conditions, if the diffusion coefficient $D$ is a constant, which means under a fixed $C$, there will be a square relation between $C$ and diffusion distance $x$. The error function solution of the diffusion equation can be described as:

$$
\operatorname{erf}\left(\frac{x}{2 \sqrt{D t}}\right)=1-\frac{2 C}{C_{0}}
$$

So under the special diffusion condition in this paper, the error function solution simplified as $\operatorname{erf}(Z)=1-2 C$. According to the diffusion concentration and distance of $\mathrm{Ni}$ in nanocrystalline and coarse-grained $\mathrm{Fe}$, the value of $\operatorname{erf}(Z)$ under different diffusion distance can be calculated. In the Gauss error function

\begin{tabular}{|c|c|c|c|c|c|c|c|}
\hline \multirow{2}{*}{ Coarse grained $\mathrm{Fe}$} & Diffusion distance/ $\mu \mathrm{m}$ & 0.5 & 1 & 1.5 & 2 & 3 & 4 \\
\hline & $Z$ (Constant pressure) & 0.185 & 0.361 & 0.818 & 1.170 & 1.324 & 1.673 \\
\hline \multirow{3}{*}{ Nano-Fe } & Diffusion distance/ $\mu \mathrm{m}$ & 2 & 4 & 6 & 8 & 10 & 12 \\
\hline & $Z$ (Constant pressure) & 0.033 & 0.350 & 0.695 & 0.950 & 1.222 & 1.652 \\
\hline & $Z$ (Pulse pressure) & 0.015 & 0.205 & 0.565 & 0.761 & 1.145 & 1.580 \\
\hline
\end{tabular}
table, the $Z$ value at different diffusion distance $(x)$ of the samples

Table $2 Z$ value of nano Fe under different diffusion treatments 
after diffusion treatment was calculated by an interpolation method, as shown in Table 2.

The equation $\operatorname{erf}\left[\frac{x}{2 \sqrt{D t}}\right]$ is Gaussian function, if order

$$
Z=\frac{x}{2 \sqrt{D t}}
$$

where, $x$ is the diffusion distance of nickel in nanocrystalline and coarse-grained Fe after different diffusion treatment, $D$ is diffusion coefficient and $t$ is diffusion time. According to Eq.(4), $Z$ and $x$ have a linear relationship and the proportional coefficient $k$ can be described as:

$$
k=\frac{1}{2 \sqrt{D t}}
$$

Fig.6 shows the relationship of diffusion distance $x$ and $Z$ of different samples after diffusion treatments. Linear fitting reveals that $x$ and $Z$ have a good linear relationship in both coarse grained and nanocrystalline $\mathrm{Fe}$, which means the diffusion of Ni in nanocrystalline also meets the Fick's law. The slope of the curves is $k$ described in Eq.(5).

When getting the $k$ value at a certain temperature, the average diffusion coefficient $D$ can be expressed as

$$
D=\frac{1}{4 k^{2} t}
$$

By linear fitting of the $x$ and $Z$ we can see that either in nanocrystalline or in coarse-grained $\mathrm{Fe}, x$ and $Z$ have a good linear relationship. The diffusion coefficients of $\mathrm{Ni}$ in coarse-grained $\mathrm{Fe}$ under constant pressure, nano-Fe under constant pressure and nano-Fe under pulse pressure were calculated by Eq.(5), as shown in Table 3 .

\subsection{Diffusion mechanisms}

The measured results show the diffusivity of $\mathrm{Ni}$ in the nanocrystalline $\mathrm{Fe}$ is larger than that in the coarse-grained $\mathrm{Fe}$. This may be attributed to the following three reasons: Firstly the pressure exerted on the diffusion couple can help the diffusion source to contact with each other within atomic scale, which makes the diffusion of atoms possible. The movement of atoms in solids is different that from in gas, which can move randomly in a relatively long distance. So it is very necessary to exert a

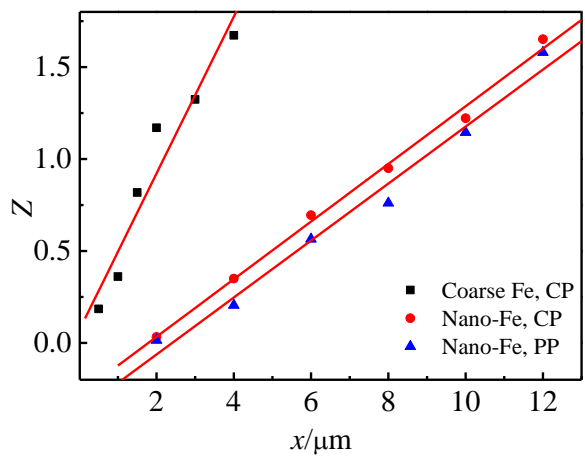

Fig.6 Linear fitting of $Z$ vs $x$ for samples after diffusion treatment at $850^{\circ} \mathrm{C}$
Table 3 Diffusion coefficient of $\mathrm{Ni}$ in different samples after diffusion treatment at $850{ }^{\circ} \mathrm{C}\left(\mathrm{m}^{2} / \mathrm{s}\right)$

\begin{tabular}{cll}
\hline Sample & Pressure & Value \\
\hline \multirow{2}{*}{ Coarse-grained Fe } & Constant pressure & $1.15 \times 10^{-15}$ \\
& Constant pressure & $8.48 \times 10^{-14}$ \\
\hline Nano-Fe & Pulse pressure & $8.69 \times 10^{-13}$ \\
\hline
\end{tabular}

pressure on the diffusion couple to make them contact with each other closely. The assembly of diffusion samples we designed can not only make the diffusion couple contact closely but also produce a certain strain. The strain can activate the nickel atoms, which is equivalent to reduce the activation energy of diffusion of the nickel atoms. This to some extent can also enhance the diffusivity of nickel atoms. Secondly, the ultrafine-grained structure provides a considerable volume fraction of GBs, which may serve as numerous fast diffusion channels for $\mathrm{Ni}$. In addition, with the decrease of the grain size of nanocrystalline $\mathrm{Fe}$, the volume fraction of triple junctions increases. These triple junctions possess a large excess volume compared to conventional GBs, which make the Ni diffusion easier. So the enhancement of the diffusivity of $\mathrm{Ni}$ in the nanocrystalline $\mathrm{Fe}$ can be attributed to both the non-equilibrium GBs and the triple junctions. Thirdly, the microstructure characteristics of the nanocrystalline Fe processed by HESP technique may be also attributed to the enhancement of the diffusivity of $\mathrm{Ni}$ in it. The severe plastic deformation at high strain occurs in the $\mathrm{Fe}$ samples during the HESP processing, which lead to the formation of an extremely high density of dislocations. These dislocations are actually in non-equilibrium which mean there are a higher stored energy and a higher density of defects than that in coarse-grained Fe. The non-equilibrium dislocations in nanocrystalline $\mathrm{Fe}$ has a higher Gibbs free energy than the conventional coarse-grained $\mathrm{Fe}$, which can also facilitate $\mathrm{Ni}$ diffusion.

The diffusivity of $\mathrm{Ni}$ in the nanocrystalline Fe under pulse pressure is also larger than that in the nanocrystalline Fe under constant pressure. According to the diffusion theory, if we want one atom to diffuse into another lattice, the atom must firstly separate from the bondage of its own lattice. So if a pressure, especially a pulse pressure exerted on the diffusion couples will produce a high alternating microstrain in the diffusion source, it can make the nickel atoms separate from their lattice easier. The diffusion of the same atom in compound diffusion (reaction diffusion) is more difficult than in solid solution (diffusion in lattice). However it is very easy for metallic compounds to form on the interface of the diffusion couple, which will change the diffusion from diffusion in lattice into reaction diffusion. The pulse pressure can break the metallic compounds formed on the interface of diffusion couple ${ }^{[11,12]}$, which can keep the diffusion of nickel in nanocrystalline $\mathrm{Fe}$ in the state of lattice diffusion. This to some extent enhances the diffusivity of $\mathrm{Ni}$ in the nanocry- 
stalline Fe compared with using constant pressure.

\section{Conclusions}

1) By HESP technique, a nanocrystalline Fe layer without contamination and porosity is fabricated on a pure iron cylinder. The average grain size at the top surface of the layer is from $58.5 \mathrm{~nm}$ to $21.2 \mathrm{~nm}$ after different shot time with a thickness from $127 \mu \mathrm{m}$ to $145 \mu \mathrm{m}$.

2) The enhanced diffusivity of $\mathrm{Ni}$ in the nanocrystalline $\mathrm{Fe}$ can be attributed to a large volume fraction of grain boundaries and a pressure exerted on the diffusion couple.

3) The grain boundaries of the nanocrystalline Fe contains a high density of dislocations and a considerable amount of triple junctions acting as diffusion channels, which make the diffusion of nickel atoms easier.

4) The pressure, especially the pulse pressure, exerted on the diffusion couple can help nickel atoms to separate from the atomic lattice, which means a reduction in diffusion activation energy of nickel atoms. The pulse pressure can also break the intermetallic compound formed on the interface of the diffusion couple, which may block the further diffusion of nickel atoms in nanocrystalline Fe.

\section{References}

1 Tao N R, Wang Z B, Tong W P et al. Acta Materialia[J], 2002, 50: 4603

2 Tao Nairong, Zhang Hongwang, Lu Jian et al. Mater Trans[J], 2003, 44(10): 1919

3 Zhu K Y, Vassel A, Brisset F et al. Acta Materialia[J], 2004, 52(14): 4101

4 Wang Z B, Lu J, Lu K. Acta Materialia[J], 2005, 53(4): 2081

5 Wang Z B, Tao N R, Tong W P et al. Acta Materialia[J], 2003, 51(14): 4319

6 Horvath J, Birringer R, Gleiter H. Solid State Communications [J], 1987, 62(5): 319

7 Kolobov Yu R, Grabovetskaya G P, Lvanov M B et al. Scripta Materialia[J], 2001, 44(6): 873

8 Wang Z B, Lu J. Acta Materialia[J], 2005, 53: 2081

9 Wang Z B, Tao N R et al. Acta Materialia[J], 2003, 51(3): 4319

10 Bei Duohui, Gu Jianfeng, Pan Jiansheng. J Mater Sci Technol. [J], 2002, 18(6): 566

11 Han J, Sheng G M, Zhou X L et al. ISIJ International[J], 2009, 49(1): 86

12 Han J, Sheng G M, Zhou X L. ISIJ International[J], 2008, 48(9): 1

\title{
应变诱导 $\mathrm{Ni}$ 原子在纳米晶 $\mathrm{Fe}$ 中的扩散行为研究
}

\author{
孙建春, 陈登明, 马毅龙 \\ (重庆科技学院, 重庆 401331)
}

\begin{abstract}
摘 要: 利用高能喷丸法 (HESP) 在工业纯铁表面制备了一定厚度无污染、空位、杂质等缺陷的纳米晶层, 其平均晶粒尺寸为 $50 \mathrm{~nm}$, 厚度为 $100 \mu \mathrm{m}$ 。在 Gleeble 1500 热模拟机上, 采用脉冲加压扩散(PPD)和恒定加压扩散(CPD)对纳米晶铁在 $850^{\circ} \mathrm{C}$ 下进行渗镍处理。结果 表明, 镍原子在纳米晶铁中的扩散系数比常规粗晶中高 1 个数量级; 而采用脉冲加压扩散时, 镍原子的扩散系数要比采用恒压扩散高 1 个数量级。镍原子扩散系数的提高主要包括三方面的原因, 一是脉冲加压能击碎扩撒界面上形成的阻碍镍原子扩散的金属件化合物; 二 是纳米晶铁中形成的大量三叉晶界; 三是纳米晶铁中形成大量的非平衡晶界。
\end{abstract}

关键词: 纳米晶铁; 高能喷丸; 扩散系数; 镍原子

作者简介：孙建春，男，1977 年生，博士，副教授，重庆科技学院冶金与材料工程学院，重庆 401331，电话：023-65023714，E-mail: kwensun@163.com 